International Mathematical Forum, 2, 2007, no. 53, 2645 - 2654

\title{
Bounds for the Relative Change of Singular Values of a Real Matrix
}

\author{
Mohammedi R. Abdel-Aziz ${ }^{1}$ \\ Department of Mathematics and Computer Science \\ Faculty of Science, Kuwait University \\ P. O. Box 5969, Safat 13060, Kuwait \\ mraziz@mcs.sci.kuniv.edu.kw
}

\begin{abstract}
This paper amis to derive bounds for the relative change in the singular values of a real matrix. Both the full column rank and the full row rank cases are considered. Two numerical experiments show that the introduced bounds are confirmed and their values are dependent on the element-wise perturbation of the matrix.
\end{abstract}

Mathematics Subject Classification: Primary 65F15; Secondary 65G05

Keywords: singular values, relative change

\section{Introduction}

The importance of relative change for the singular values arises in deflation criteria to break the problem up into subproblems. This will enhance the efficiency and will make the subproblems suitable for the design of accurate parallel divided-and-conquer algorithms. Deflation techniques are needed in large scale problems of structural eigensystems $[7,1]$.

In this paper, we consider $A \in R^{m \times n}$ and study both cases of full column rank and row rank [6]. When $m \geq n$ and $\operatorname{rank}(A)=n$ (full column rank), the singular value decomposition (SVD) of $A$ is given by

$$
A=U\left(\begin{array}{c}
\Sigma \\
O
\end{array}\right) V^{T},
$$

\footnotetext{
${ }^{1}$ On leave from Department of Mathematics, Faculty of Science, Alexandria University, Egypt.
} 
where $U \in R^{m \times m}$ and $V \in R^{n \times n}$ are orthogonal and $\Sigma=\operatorname{diag}\left(\sigma_{1}, \sigma_{2}, \cdots, \sigma_{n}\right)$ and $\sigma_{i}>0$ for $1 \leq i \leq n$. In the complex case, the matrices $U$ and $V$ are unitary. The eigenvalue decomposition corresponding to (1.1) is

$$
A^{T} A=V\left(\begin{array}{c}
\Lambda \\
O
\end{array}\right) V^{T}, \quad \Lambda=\Sigma^{2}
$$

The introduced class of perturbations consists of all $\Delta A$ for which $A+\Delta A$ is scaled from the right hand side. In the context of the SVD of a real matrix $A$, we consider the perturbation $A+\Delta A=(C+\Delta C) B$ for $n \times n$ nonsingular matrices $B$. This class of perturbation include component-wise relative perturbations. The addressed problem has been studied by some researchers in $[8,11]$. Our investigation is completely different because the introduced bounds are distinguished and confirmed numerically.

This work is organized as follows. In Section 2, we recall some lemmas from [8] and prove some others which are the basis for finding our bounds. Section 3 involves bounds for the full column rank case for the right side scaling. Section 4 is devoted to introducing bounds for the row rank case. Section 5 contains some numerical experiments that illustrate and confirm the investigated bounds. Finally, concluding remarks and further ideas are given in Section 6.

\section{Preliminaries}

Throughout this section, we recall some lemmas without proofs and prove some others. These lemmas will be considered as a basis for our results.

Definition 1 An $n \times n$ real matrix $S$ is doubly stochastic matrix if all $s_{i j} \geq 0$ and

$$
\sum_{k=1}^{n} s_{k j}=\sum_{k=1}^{n} s_{i k}=1, \text { for all } 1 \leq i, j \leq n .
$$

For more details about stochastic matrices and some of their properties, we refer the reader to ref. [12].

Lemma 2.1 Let $S$ be an $n \times n$ doubly stochastic matrix and $Q$ be an $n \times n$ real matrix. Then there exists a permutation $\pi$ of $\{1,2, \cdots, n\}$ such that

$$
\sum_{i=1}^{n}\left|Q_{i \pi(i)}\right|^{2} \leq \sum_{i, j=1}^{n}\left|Q_{i j}\right|^{2} S_{i j}
$$

Proof: See ref. [4]. 
Lemma 2.2 Let $\left\{\mu_{1}, \mu_{2}, \cdots, \mu_{n}\right\}$ and $\left\{\lambda_{1}, \lambda_{1}, \cdots, \lambda_{n}\right\}$ be two sets of positive real numbers. Then there exists a permutation $\pi$ of $\{1,2, \cdots, n\}$ such that

$$
\sum_{i=1}^{n} \frac{\left(\mu_{i}-\lambda_{i}\right)^{2}}{\left|\mu_{i} \lambda_{i}\right|}=\min _{\pi} \sum_{i=1}^{n} \frac{\left(\mu_{i}-\lambda_{\pi(i)}\right)^{2}}{\left|\mu_{i} \lambda_{\pi(i)}\right|}
$$

Proof: See ref. [8].

Lemma 2.3 Let $\alpha$ and $\beta$ be two real numbers. Then $\frac{(\alpha+\beta)}{\sqrt{\alpha \beta}} \geq 2$.

Proof: The proof is completed by using the inequality $(\sqrt{\alpha}-\sqrt{\beta})^{2} \geq 0$.

Lemma 2.4 Let $\alpha$ and $\beta$ be two positive real numbers. Then $\frac{|\alpha-\beta|}{\sqrt{\alpha \beta}} \leq$ $\frac{1}{2}\left(\frac{\left|\alpha^{2}-\beta^{2}\right|}{\alpha \beta}\right)$

Proof: The right hand side of the lemma can be written as

$$
\frac{\left|\alpha^{2}-\beta^{2}\right|}{\alpha \beta}=\frac{|\alpha-\beta|}{\sqrt{\alpha \beta}} \frac{|\alpha+\beta|}{\sqrt{\alpha \beta}} .
$$

The proof is completed by substituting from Lemma (2.3) into (2.1).

Lemma 2.5 Let $0<\alpha \leq \beta \leq \xi \leq \eta$. Then $\quad \frac{\eta-\alpha}{\sqrt{\alpha \eta}} \geq \frac{\xi-\beta}{\sqrt{\beta \xi}}$

Proof: From the given assumption, we can write $0<\frac{\alpha}{\eta} \leq \frac{\beta}{\xi} \leq 1$. Thus, we obtain

$$
\frac{|\eta-\alpha|}{\sqrt{\alpha \eta}}=\frac{\eta}{\sqrt{\alpha \eta}}-\frac{\alpha}{\sqrt{\alpha \eta}}=\frac{1}{\sqrt{\alpha / \eta}}-\sqrt{\alpha / \eta} \geq \frac{1}{\sqrt{\beta / \xi}}-\sqrt{\beta / \xi}=\frac{\xi-\beta}{\sqrt{\beta \xi}}
$$

which completes the proof.

Lemma 2.6 Let $D$ be an $n \times n$ matrix such that $\|D\|_{2}<1$. Then

$$
\left\|(I+D)^{-\frac{1}{2}} D\right\|_{F} \leq \frac{\|D\|_{F}}{\sqrt{1-\|D\|_{2}}}
$$

Proof: From the properties of norms, we can write

$$
\left\|(I+D)^{-\frac{1}{2}} D\right\|_{F} \leq\left\|(I+D)^{-\frac{1}{2}}\right\|_{F}\|D\|_{F} .
$$

But, for any $n \times n$ matrix $A$, we have $\|A\|_{2} \leq\|A\|_{F}$. Thus,

$$
\left\|(I+A)^{-\frac{1}{2}}\right\|_{F} \leq \frac{1}{\sqrt{1-\|A\|_{2}}} .
$$

The proof is completed by introducing inequality (2.2) into inequality (2.3), for more details about these inequalities, we refer the reader to [12]. 


\section{Bounds for the Full Column Rank Case}

Throughout this section, we consider $A$ and $\tilde{A}=A+\Delta A$ are real $m \times n$ matrices such that $m \geq n$ and one is the perturbation of the other. Denote their singular values by

$$
\sigma(A)=\left\{\sigma_{1}, \sigma_{2}, \cdots, \sigma_{n}\right\} \text { and } \sigma(\tilde{A})=\left\{\rho_{1}, \rho_{2}, \cdots, \rho_{n}\right\}
$$

and they are ordered so that

$$
\sigma_{1} \geq \sigma_{2} \geq \cdots \geq \sigma_{n}>0 \text { and } \rho_{1} \geq \rho_{2} \geq \cdots \geq \rho_{n}>0
$$

Here, we consider $A$ scaled from the right hand side. Let $A=C B$ and $\triangle A=$ $\triangle C B$, where $B$ is an $n \times n$ nonsingular matrix. Then, we have

$$
\tilde{A}=A+\Delta A=(C+\Delta C) B=\left(I+\Delta C C^{\dagger}\right) A=(I+D) A,
$$

where $D=\Delta C C^{\dagger}$. We set $\eta=\|D\|_{F}$ and $\xi=\|D\|_{2}$.

Lemma 3.1 Let $A, \tilde{A}$ and $\Delta A$ be as defined before, then the singular values $\sigma_{i}$ and $\rho_{i}$ that are ordered as in (3.2) satisfy the inequality $\left(\sum_{i=1}^{n}\left(\frac{\sigma_{i}^{2}-\rho_{i}^{2}}{\sigma_{i} \rho_{i}}\right)^{2}\right)^{\frac{1}{2}} \leq\|\Gamma\|_{F}$, where

$\Gamma=U^{T}\left(D^{T}+D+D^{T} D\right)\left(I+D^{T}+D+D^{T} D\right)^{-\frac{1}{2}} \tilde{U}$.

Proof: Let $G=A^{T} A, \tilde{G}=G+\Delta G=\tilde{A}^{T} \tilde{A}$ and applying formula (3.3), we obtain

$$
\tilde{G}=A^{T}(I+D)^{T}(I+D) A=A^{T}\left(I+D^{T}+D+D^{T} D\right) A .
$$

Setting $\tilde{A}=\left(I+D^{T}+D+D^{T} D\right)^{\frac{1}{2}} A$ and taking in account that $\tilde{G} \tilde{X}=$ $(G+\Delta G) \tilde{X}=\tilde{X} \tilde{\Lambda}$ and $G X=X \Lambda$, we obtain $X^{T}(G+\Delta G) \tilde{X}=X^{T} \tilde{X} \tilde{\Lambda}$ which implies $X^{T} G \tilde{X}+X^{T} \Delta G \tilde{X}=X^{T} \tilde{X} \tilde{\Lambda}$. Thus,

$$
\Lambda X^{T} \tilde{X}-X^{T} \tilde{X} \tilde{\Lambda}=-X^{T} \Delta G \tilde{X}
$$

From formula (3.4), we obtain

$$
\begin{aligned}
X^{T} \Delta G \tilde{X} & =X^{T}(\tilde{G}-G) X=X^{T} A^{T}\left(D^{T}+D+D^{T} D\right) A \tilde{X} \\
& =X^{T} A^{T}\left(D^{T}+D+D^{T} D\right)\left(I+D^{T}+D+D^{T} D\right)^{-\frac{1}{2}}\left(I+D^{T}+D+D^{T} D\right)^{\frac{1}{2}} A \tilde{X} \\
& =X^{T} A^{T}\left(D^{T}+D+D^{T} D\right)\left(I+D^{T}+D+D^{T} D\right)^{-\frac{1}{2}} \tilde{A} \tilde{X} .
\end{aligned}
$$

Since, $\tilde{G} \tilde{X}=\tilde{X} \tilde{\Lambda}$ and $G X=X \Lambda$ imply that there exists an orthogonal matrix $\tilde{U}$ such that $\tilde{A} \tilde{X}=\tilde{U} \tilde{\Lambda}^{\frac{1}{2}}$. Similarly, there exists an orthogonal matrix $U$ such 
that $A X=U \Lambda^{\frac{1}{2}}$. Setting $\Gamma=U^{T}\left(D^{T}+D+D^{T} D\right)\left(I+D^{T}+D+D^{T} D\right)^{-\frac{1}{2}} \tilde{U}$. Hence, formula (3.6) becomes

$$
X^{T} \Delta G \tilde{X}=\Lambda^{\frac{1}{2}} \Gamma \tilde{\Lambda}^{\frac{1}{2}}
$$

From (3.7) in (3.5), we obtain

$$
\Lambda X^{T} \tilde{X}-X^{T} \tilde{X} \tilde{\Lambda}=-\Lambda^{\frac{1}{2}} \Gamma \tilde{\Lambda}^{\frac{1}{2}}
$$

Using equality (3.8) component wise for all pairs of indices $(i, j)$, squaring each term, adding them all together and setting $\left|S_{i j}\right|=\left|\left(X^{T} \tilde{X}\right)_{i j}\right|$, we obtain

$$
\sum_{i, j=1}^{n}\left(\frac{\Lambda_{i i}-\tilde{\Lambda}_{j j}}{\sqrt{\left|\Lambda_{i i} \tilde{\Lambda}_{j j}\right|}}\right)^{2}\left|S_{i j}\right|^{2}=\|\Gamma\|_{F}^{2}
$$

Taking in account that the matrix $\left(\left|S_{i j}\right|\right)$ is doubly stochastic and applying Lemma 2.1, we obtain

$$
\sum_{i=1}^{n}\left(\frac{\Lambda_{i i}-\tilde{\Lambda}_{\pi(i) \pi(i)}}{\sqrt{\left|\Lambda_{i i} \tilde{\Lambda}_{\pi(i) \pi(i)}\right|}}\right)^{2} \leq \sum_{i, j=1}^{n}\left(\frac{\Lambda_{i i}-\tilde{\Lambda}_{j j}}{\sqrt{\left|\Lambda_{i i} \tilde{\Lambda}_{j j}\right|}}\right)^{2}\left|S_{i j}\right|^{2}
$$

Applying Lemma 2.2 on the left hand side of inequality (3.10), this inequality becomes

$$
\sum_{i=1}^{n}\left(\frac{\Lambda_{i i}-\tilde{\Lambda}_{i i}}{\sqrt{\left|\Lambda_{i i} \tilde{\Lambda}_{i i}\right|}}\right)^{2} \leq \sum_{i=1}^{n}\left(\frac{\Lambda_{i i}-\tilde{\Lambda}_{\pi(i) \pi(i)}}{\sqrt{\left|\Lambda_{i i} \tilde{\Lambda}_{\pi(i) \pi(i)}\right|}}\right)^{2} .
$$

The proof is completed by introducing inequalities (3.9) and (3.10) into inequality (3.11), taking the square root of both sides and using the fact that $\Lambda_{i i}=\sigma_{i}^{2}$ and $\widetilde{\Lambda}_{i i}=\rho_{i i}^{2}$.

Lemma 3.2 Let the assumptions of Lemma 3.1 hold. Then

$$
\left(\sum_{i=1}^{n}\left(\frac{\left|\sigma_{i}-\rho_{i}\right|}{\max \left(\sigma_{i} \rho_{i}\right)}\right)^{2}\right)^{\frac{1}{2}} \leq \frac{2 \eta+\eta^{2}}{\sqrt{1-2 \xi-\xi^{2}}}
$$

Proof: Setting $\Delta M=D^{T}+D+D^{T} D$ in the definition of $\Gamma$, we obtain $\Gamma=U^{T} \Delta M(I+\Delta M)^{-\frac{1}{2}} \tilde{U}$. Applying Lemma 2.6, we obtain

$$
\|\Gamma\|_{F} \leq \frac{\|\Delta M\|_{F}}{\sqrt{1-\|\Delta M\|}}
$$


Using definition of $\|D\|_{F}$ and $\|D\|$, we obtain $\|\Delta M\|_{F} \leq 2 \eta+\eta^{2}$ and $\|\Delta M\| \leq$ $2 \xi+\xi^{2}$. Thus, inequality (3.12) becomes

$$
\|\Gamma\|_{F} \leq \frac{2 \eta+\eta^{2}}{\sqrt{1-2 \xi-\xi^{2}}} .
$$

Also, we have

$$
\frac{\left|\sigma_{i}-\rho_{i}\right|}{\max \left(\sigma_{i} \rho_{i}\right)} \leq \frac{\sigma_{i}^{2}-\rho_{i}^{2}}{\left[\max \left(\sigma_{i} \rho_{i}\right)\right]^{2}} \leq \frac{\sigma_{i}^{2}-\rho_{i}^{2}}{\sigma_{i} \rho_{i}} .
$$

The proof is completed by inserting inequalities (3.13) and (3.14) in Lemma 3.1 .

\section{Bounds for the Full row rank case}

The full row rank case is more complicated than the full column rank case. Let $Q$ be real $m \times n$ matrix with full row rank, $\operatorname{rank}(Q)=m \leq n$. The $\operatorname{SVD}$ for $Q$ can be written as $Q=U[\Sigma O] V^{T}$, where $U$ and $V$ are unitary matrices, $\Sigma=$ $\operatorname{diag}\left\{\mu_{1}, \mu_{2}, \cdots, \mu_{m}\right\}$ and $\mu_{i}>0$. The corresponding nonsingular eigenvalue problem can be written as $Q Q^{T}=U \Lambda U^{T}$, where $\Lambda=\Sigma^{2}$. This eigenproblem will take the form

$$
V^{T} Q^{T} Q V=\left[\begin{array}{cc}
|\Lambda| & O \\
O & O
\end{array}\right]
$$

where $V^{T} V=I$ and $O$ is the zero matrix. Let us partition $V$ as $V=\left[\begin{array}{ll}V_{0} & V_{1}\end{array}\right]$, where $V_{0}=V(:, 1: m)$. Then for all $V$ which perform (4.1), the parts $V_{0}$ have the same spectral norm [13]. Clearly if $V$ performs (4.1), then any $\mathcal{V}$ of the form

$$
\mathcal{V}=V\left[\begin{array}{cc}
I & O \\
O & \mathcal{V}_{1}
\end{array}\right]=\left[\begin{array}{ll}
V_{0} & V_{1} \mathcal{V}_{1}
\end{array}\right]
$$

when $\mathcal{V}_{1}^{T} \mathcal{V}_{1}=I$ does the same.

Let $Q=P R$, where $P$ is an $m \times m$ nonsingular matrix. For then the perturbation $\Delta Q=P \Delta R$. We have

$$
\tilde{Q}=Q+\Delta Q=Q\left(I+Q^{\dagger} \Delta Q\right)=P R\left(I+R^{\dagger} \Delta R\right) .
$$

For simplicity, set $W=R^{\dagger} \Delta R, \beta_{1}=\|W\|_{2}$ and $\beta_{2}=\|W\|_{F}$.

Lemma 4.1 Let $Q, \tilde{Q}, \Delta Q, W, \beta_{2}$ and $\beta_{1}$ be as defined before, respectively. Let the $S V D$ of $\tilde{Q}$ given by $\tilde{Q}=\tilde{U}[\tilde{\Sigma} O] \tilde{V}^{T}$, where $\tilde{\Sigma}=\operatorname{diag}\left\{\tilde{\mu}_{1}, \tilde{\mu}_{2}, \cdots, \tilde{\mu}_{m}\right\}$, $\tilde{\mu}_{i}>0$ and let $\tilde{V}=\left[\begin{array}{ll}\tilde{V}_{0} & \tilde{V}_{1}\end{array}\right]$. If $\beta_{1}<1$, then the unperturbed and perturbed singular values $\mu_{i}$ and $\tilde{\mu}_{i}$, respectively satisfy the inequality

$$
\left(\sum_{i=1}^{m}\left(\frac{\mu_{i}^{2}-\tilde{\mu}_{i}^{2}}{\mu_{i} \tilde{\mu}_{i}}\right)^{2}\right)^{\frac{1}{2}} \leq\left\|V_{0}\right\|\left\|\tilde{V}_{0}\right\| \frac{2 \beta_{2}+\beta_{2}^{2}}{1-\beta_{1}}
$$


Proof: Let $K=U \Lambda U^{T}$ and $\tilde{K}=\tilde{U} \tilde{\Lambda} \tilde{U}^{T}$ be the corresponding unperturbed and perturbed eigenproblems, where $K=Q Q^{T}$ and $\tilde{K}=\tilde{Q} \tilde{Q}^{T}$. Then $\tilde{K}=$ $Q(I+W)(I+W)^{T} Q^{T}$ with $\Delta K=\tilde{K}-K$. Thus, we can write

$$
\tilde{K}-K=\tilde{Q}\left[(I+W)^{T}-(I+W)^{-1}\right] Q^{T}=\tilde{Q} \mathcal{H} Q^{T},
$$

where $\mathcal{H}=\left[(I+W)^{T}-(I+W)^{-1}\right]$. Multiplying (4.2) from the left by $\tilde{U}^{T}$ and from the right by $U$ and using the corresponding eigenproblems, we obtain

$$
\tilde{U}^{T} \tilde{Q} \mathcal{H} Q^{T} U=\tilde{U}^{T}[\tilde{K}-K] U=\tilde{U}^{T}\left[\tilde{U} \tilde{\Lambda} \tilde{U}^{T}-U \Lambda U^{T}\right] U=\tilde{\Lambda} \tilde{U}^{T} U-\tilde{U}^{T} U \Lambda
$$

From before, we have

$$
U^{T} Q=[\Sigma O] V^{T}=\Sigma V_{0}^{T}
$$

Combining (4.3) and (4.4), we obtain

$$
\tilde{\Lambda} \tilde{U}^{T} U-\tilde{U}^{T} U \Lambda=\left|\tilde{\Lambda}^{\frac{1}{2}}\right| \tilde{V}_{0}^{T} \mathcal{H} V_{0}\left|\Lambda^{\frac{1}{2}}\right|
$$

In formula (4.5), taking component-wise for all pairs of indices $(i, j)$, squaring each term, adding them all together and setting $\left|Z_{i j}\right|=\left|\left(\tilde{U}^{T} U\right)_{i j}\right|$, we obtain

$$
\sum_{i, j=1}^{m}\left(\frac{\left|\tilde{\mu}_{i}^{2}-\mu_{j}^{2}\right|}{\left|\tilde{\mu}_{i} \mu_{j}\right|}\right)^{2}\left|Z_{i j}\right|^{2}=\|\left.\tilde{V}_{0}^{T} \mathcal{H} V_{0}\right|_{F} ^{2}
$$

Defining the matrix $X=\left(\left|Z_{i j}\right|\right)$ and applying definition 1 , we conclude that $X$ is a doubly stochastic matrix. From Lemma 2.1, we obtain

$$
\sum_{i=1}^{m}\left(\frac{\left|\tilde{\mu}_{i}^{2}-\mu_{\pi(i)}^{2}\right|}{\left|\tilde{\mu}_{i}\right|\left|\mu_{\pi(i)}\right|}\right)^{2} \leq\left\|\tilde{V}_{0}^{T} \mathcal{H} V_{0}\right\|_{F}^{2}
$$

Applying Lemma 2.2 on the left hand side of inequality (4.7), we obtain

$$
\sum_{i=1}^{m}\left(\frac{\left|\tilde{\mu}_{i}^{2}-\mu_{j}^{2}\right|}{\left|\tilde{\mu}_{i}\right|\left|\mu_{j}\right|}\right)^{2} \leq \sum_{i=1}^{m}\left(\frac{\left|\tilde{\mu}_{i}^{2}-\mu_{\pi(i)}^{2}\right|}{\left|\tilde{\mu}_{i}\right|\left|\mu_{\pi(i)}\right|}\right)^{2} .
$$

The matrix $\mathcal{H}$ can be reformulated as

$$
\begin{aligned}
\mathcal{H} & =(I+W)^{-1}\left[(I+W)\left(I+W^{T}\right)-I\right] \\
& =(I+W)^{-1}\left[I+W^{T}+W+W W^{T}-I\right] \\
& =(I+W)^{-1}\left[W^{T}+W+W W^{T}\right] .
\end{aligned}
$$

From the definitions of norms of $W$, we obtain $\|\mathcal{H}\|_{F} \leq \frac{2 \beta_{2}+\beta_{2}^{2}}{1-\beta_{1}}$. Introducing the bound of $\|\mathcal{H}\|_{F}$ into inequality (4.7), we obtain

$$
\sum_{i=1}^{m}\left(\mid \frac{\tilde{\mu}_{i}^{2}-\mu_{\pi(i)}^{2} \mid}{\left|\tilde{\mu}_{i}\right|\left|\mu_{\pi(i)}\right|}\right)^{2} \leq\left\|\tilde{V}_{0}^{T}\right\|^{2}\left\|V_{0}\right\|^{2}\left(\frac{2 \beta_{2}+\beta_{2}^{2}}{1-\beta_{1}}\right)^{2} .
$$

The proof is completed by combining inequalities (4.9) and (4.8) and taking the square root of both sides. 


\section{$5 \quad$ Numerical Experiments}

In this section, we confirm our theoretical results on some examples. We give two examples for the component wise perturbation. Such perturbations are highly interesting since they appear during various numerical algorithms for singular values $[4,10]$. The following example illustrates the given results in lemmas 3.1 and 3.2 .

Example 1. Let $A$ and $\tilde{A}$ be two matrices with positive singular values and one is the perturbation of the other such that $A=C B, \triangle A=\triangle C B$ and $\tilde{A}=A+\Delta A$, where

$C=10^{-2} \times\left[\begin{array}{cccc}200 & 8 & 7 & 6 \\ 8 & 200 & 8 & 7 \\ 7 & 8 & 200 & 8 \\ 6 & 7 & 8 & 200 \\ 5 & 6 & 7 & 100\end{array}\right]$ and $B=10^{-2} \times\left[\begin{array}{cccc}300 & 5 & 4 & 6 \\ 5 & 300 & 8 & 7 \\ 4 & 8 & 300 & 8 \\ 3 & 6 & 7 & 300\end{array}\right]$.

The element wise perturbation matrices $\Delta C$ and $\Delta A$ are

$\Delta C=10^{-3} \times\left[\begin{array}{cccc}90 & 2 & 3 & 1 \\ 2 & 90 & 1 & 2 \\ 3 & 1 & 80 & 1 \\ 1 & 2 & 1 & 80 \\ 0 & 2 & 1 & 30\end{array}\right]$ and $\Delta A=10^{-4} \times\left[\begin{array}{cccc}2702 & 108 & 128 & 88 \\ 106 & 2703 & 104 & 125 \\ 123 & 96 & 2403 & 97 \\ 55 & 109 & 88 & 2403 \\ 10 & 79 & 53 & 902\end{array}\right]$.

The matrix $A$ and its perturbed matrix $\tilde{A}$ are given by

$A=10^{-4} \times\left[\begin{array}{cccc}60086 & 3492 & 3006 & 3112 \\ 3453 & 60146 & 4081 & 3621 \\ 2964 & 4083 & 60148 & 4098 \\ 2467 & 3394 & 3880 & 60149 \\ 1858 & 2481 & 2868 & 30128\end{array}\right]$ and $\tilde{A}=10^{-4} \times\left[\begin{array}{cccc}62788 & 3600 & 3134 & 3200 \\ 3559 & 62849 & 4185 & 3737 \\ 3087 & 4179 & 62551 & 4194 \\ 2522 & 3503 & 3968 & 62552 \\ 1868 & 2560 & 2921 & 31030\end{array}\right]$

The singular values and their squares of $A$ and $\tilde{A}$ are

\begin{tabular}{||c|c|c|c|c||}
\hline \hline & 1 & 2 & 3 & 4 \\
\hline$\sigma(A)$ & 4.8914 & 5.5994 & 5.9236 & 8.8306 \\
\hline$\sigma(\tilde{A})$ & 5.1225 & 5.8484 & 6.1873 & 9.1072 \\
\hline$\sigma^{2}(A)$ & 23.9261 & 31.3535 & 35.0894 & 77.9799 \\
\hline$\sigma^{2}(\tilde{A})$ & 26.2403 & 24.2041 & 38.2828 & 82.9402 \\
\hline
\end{tabular}

Applying lemmas 3.1 and 3.2, the left-hand sides of these lemmas are 0.1651 and 0.0825 . The bounds on the right-hand sides are 0.1654 and 0.1851 , respectively. The matrix of perturbation $\Delta C$ plays a crucial role in the computations. 
If the elements of this matrix are very small, then the quantity $\sqrt{1-2 \xi-\xi^{2}}$ will be pure imaginary.

Our second example illustrates bounds for the full row rank case.

Example 2. Let $R=10^{-2} \times\left[\begin{array}{ccccc}150 & 9 & 8 & 7 & 5 \\ 9 & 200 & 8 & 7 & 6 \\ 8 & 8 & 200 & 8 & 7 \\ 7 & 7 & 8 & 150 & 300\end{array}\right], Q=P R$ and $\Delta Q=P(0.02 R)$. Thus, if

$P=10^{-2} \times\left[\begin{array}{cccc}30 & 5 & 4 & 6 \\ 5 & 30 & 8 & 7 \\ 4 & 8 & 30 & 8 \\ 3 & 6 & 7 & 30\end{array}\right]$, then $\Delta Q=10^{-4} \times\left[\begin{array}{ccccc}92 & 27 & 23 & 36 & 40 \\ 23 & 123 & 39 & 76 & 47 \\ 19 & 39 & 123 & 84 & 54 \\ 15 & 30 & 34 & 11 & 182\end{array}\right]$,

the matrix $W=R^{\dagger}(0.02 R)$ and the perturbed matrix $\tilde{Q}=Q(I+W)$, where $\Delta Q=Q W$ are given by

$$
W=10^{-4} \times\left[\begin{array}{ccccc}
200 & 0 & 0 & -1 & 1 \\
0 & 186 & -16 & 44 & -21 \\
0 & -16 & 181 & 50 & -24 \\
-1 & 44 & 50 & 64 & 66 \\
1 & -21 & -24 & 66 & 168
\end{array}\right], \tilde{Q}=10^{-4} \times\left[\begin{array}{ccccc}
4711 & 1371 & 1151 & 1816 & 2048 \\
1156 & 6281 & 1975 & 3902 & 2408 \\
0987 & 1971 & 6283 & 4272 & 2732 \\
0785 & 1523 & 1746 & 5611 & 9282
\end{array}\right]
$$

The squares of singular values of $Q$ and $\tilde{Q}$ are

\begin{tabular}{||c|c|c|c|c||}
\hline \hline & 1 & 2 & 3 & 4 \\
\hline$\mu^{2}$ & 0.1554 & 0.1841 & 0.3315 & 2.1382 \\
\hline$\tilde{\mu}^{2}$ & 0.1617 & 0.1916 & 0.3449 & 2.2246 \\
\hline
\end{tabular}

The values of $\|W\|_{2}$ and $\|W\|_{F}$ are 0.02 and 0.04 , respectively. Applying Lemma 4.1, the left-hand side of this lemma is 0.0792 and the bound on the right-hand side is 0.0833 .

\section{Concluding Remarks}

We have introduced some bounds for singular values for the full column rank and the full row rank problems. We have investigated the effect of perturbations in singular values when the elements of the matrix are perturbed. This investigation is sufficiently general that holds for any problem scaled from the right hand side. The first question which naturally arises is: what happens if the elements of the matrix are complex numbers or they are dependent on a parameter $\gamma$ ?

Finally, a further possible problem to look at, in continuation of the present results is: what about the perturbation theory for the quadratic eigenpblem $\left(\lambda^{2} A+\lambda B+C\right) x=0 ?$ 


\section{References}

[1] M. R. Abdel-Aziz, Safeguarded use of the implicitly restarted Lanczos technique for solving non-linear structural eigensystems, International Journal for Numerical Methods in Engineering, 37(1994), 3117-3133.

[2] J. Demmel and K. Veselic, Jacobi's method is more accurate than $Q R$, SIAM Journal of Matrix Analysis and its Applications, 13(1992) 12041245 .

[3] S. C. Eisenstat and I. C. F. Ipsen, Three absolute perturbation bounds for matrix eigenvalues imply relative bounds, SIAM J. of Matrix Anal. Appl., 20(1998), 149-158.

[4] L. Elsner and S. Friedland, Singular values, doubly stochastic matrices, Journal of Linear Algebra and Applications, 220(1995), 161-169.

[5] G. H. Golub and C. F. Van Loan, Matrix Computations, 3rd. ed., Baltimore: The Johns Hopkins University Press, 1996.

[6] R. A. Horn and C. R. Johnson, Topics in Matrix Analysis, New York: Cambridge University Press, 1991.

[7] R. B. Lehoucq and D. C. Sorensen, Deflation techniques for an implicitly restarted Arnoldi iteration, SIAM J. of Matrix Analysis and its Applications., 17(1996), 789-821.

[8] R.-C. Li, Relative perturbation theory I: eigenvalue and singular value variations, SIAM J. of Matrix Analysis and its Applications, 19(1998), 956-982.

[9] R. Mathias, Spectral perturbation bounds for positive definite matrices, SIAM J. of Matrix Analysis and its Applications, 18(1997), 959-980.

[10] I. Slapincăr and N. Truhar, Relative perturbation theory for hyperbolic singular value problem, J. of Linear Algebra and Applications, 358(2003), $367-386$.

[11] M. Stewart, Perturbation of the SVD in the presence of small singular values, J. of Linear Algebra and Applications, 419(2006), 53-77.

[12] G. W. Stewart and J. Sun, Matrix Perturbation Theory, Academic Press Limited, London, 1990.

[13] K. Veselic, Perturbation theory for eigenvalues of factorized symmetric matrices, Linear Algebra and Applications, 309(2000), 85-102.

Received: January 8, 2007 